\title{
La edad y la resiliencia en el personal de salud que atiende pacientes con Covid-19. Caso de la ciudad de Ambato.
}

The age and recovery capacity of health personnel who care for patients with Covid-19. Case of the city of Ambato.

Paulina del Roció Gordón Villalba. ${ }^{1}$, Manolo Alexander Córdova Suárez. ${ }^{2}$, Edison Patricio Villacres Cevallos. ${ }^{3} \&$ Enrique Mauricio Barreno Avila. ${ }^{4}$

\section{Abstract.}

Introduction. Introduction. The COVID-19 pandemic can generate stressful events and an aggressive work environment that can influence the ability to successfully adapt to adversity or resilience of hospital health personnel who care for coronavirus patients on the front line. Objective. The psychometric properties in the health personnel of the hospitals of the city of Ambato who work directly with patients infected by

\section{Resumen.}

Introducción. La pandemia por COVID19 puede generar eventos estresantes y un ambiente de trabajo agresivo que pueden influenciar en la capacidad de adaptarse con éxito a la adversidad o resiliencia al personal sanitario de hospitales que atiende en primera línea a enfermos por corona virus. Objetivo. Se evaluó las propiedades psicométricas en el personal de salud de los hospitales de la ciudad de Ambato que laboran directamente con

\footnotetext{
1 Universidad Técnica de Ambato, Facultad de Ciencias de la Salud, Ambato-Ecuador, pdr.gordon@uta.edu.ec, iD http//orcid.org/0000-0002-1416-7560

2 Universidad Nacional de Chimborazo, Facultad de Ingeniería, Riobamba-Ecuador, hesconsultores@hotmail.com, iD http//orcid.org/0000-0001-6786-7926

3 Universidad Nacional de Chimborazo, Facultad de Ingeniería, Riobamba-Ecuador, pvillacres@ unach.edu.ec, iD http//orcid.org/0000-0001-9518-1278

4 Universidad Técnica de Ambato, Facultad de Ingeniería Civil y Mecánica, Ambato, Ecuador, enriquebarrenoavila@gmail.com, iD https://orcid.org/0000-0001-5221-7664
} 
COVID-19 were evaluated using the Connor-Davidson resilience scale (CDRISC) at different ages. Methodology. The level of: Strength, Optimism, Objectives and Resources was determined by applying a digital questionnaire of 22 questions on a scale of 5 levels of affectation: a) very low, b) low, c) medium, d) high and e) very high a sample of 70 health workers using the CD-RISC method. Finally, the incidence of age in the final value of the resilience scale by gender was verified. Results. It was determined that the highest resilience level value is for female health personnel with a value of 632 at the age of 32 , on the other hand, the highest resilience level was found for the male gender with a value from 327 to an age of 26. Likewise, the highest levels of Strength, Optimism, Objectives and Resources were determined with values of: 225, 223, 186 and 217 respectively at 32 years of age. Conclusion. The highest level of resilience for health personnel in the city of Ambato is found in the female population at the age of 32, surpassing male personnel by $51.57 \%$.

Keywords: Resilience, health, COVID19, age. pacientes infectados por COVID-19 utilizando la escala de resiliencia de Connor-Davidson (CD-RISC) a diferentes edades. Metodología. Se determinó el nivel de: Fortaleza, Optimismo, Objetivos y Recursos aplicando un cuestionario digital de 22 preguntas en una escala de 5 niveles de afectación: a) muy bajo, b) bajo, c) medio, d) alto y e) muy alto a una muestra de 70 trabajadores sanitarios utilizando el método CD-RISC. Finalmente se comprobó la incidencia de la edad en el valor final de la escala de resiliencia por género. Resultados. Se determinó que el valor más alto de nivel de resiliencia la tiene el personal sanitario de género femenino con un valor de 632 a la edad de 32 años, por otro lado, se encontró el nivel de resiliencia más alto para el género masculino con un valor de 327 a una edad de 26 años. Así mismo se determinaron los niveles más altos de Fortaleza, Optimismo, Objetivos y Recursos con valores de: 225, 223, 186 y 217 respectivamente a los 32 años. Conclusión. El nivel de resiliencia más alto para el personal sanitario de la ciudad de Ambato la tienen la población femenina a la edad de 32 años superando al personal masculino en un $51,57 \%$.

Palabras claves: Resiliencia, sanitario, COVID-19, edad.

\section{Introducción.}

La pandemia por COVID-19 causó una emergencia a nivel mundial con implicaciones severas en la salud de las personas. Las técnicas de control básicas que los países a nivel mundial emplearon desde su inicio derivaron en el del rastreo de casos, el aislamiento de los infectados y cuarentenas obligatorias. Este impedimento de trabajo presencial en los trabajos tradicionales ocasionó un quebrantamiento en el sistema financiero (Abdullahi et al. 2020), pero el sector sanitario no paró y se acomodó a este ambiente agresivo de trabajo generando posibles situaciones de exposición a elementos estresantes por el 
manejo directo de pacientes e indirecto de los sitios contaminados por este virus mortífero (Yin et al. 2020).

Las técnicas de bioseguridad que se aplican en el sector sanitario fundamentan su accionar en el uso de equipo de protección personal básico y métodos de desinfección continuos. El alejamiento del personal sanitario no es posible por el manejo del paciente lo que hace de esta condición de trabajo muy peligrosa y de alto riesgo de contagio(Cavalcanti et al. 2020). Este contacto continuo con superficies contaminadas y el incremento de fatalidades en los hospitales por el contagio masivo hacen de estos puestos de trabajo muy estresantes ya que el manejo emocional de los afectados directos e indirectos incurre en el desarrollo de habilidades para el manejo de situaciones tanto de los médicos tratantes, enfermeras y personal de soporte. Solo solo pocos tienen preparación y experiencia para afrontar estas situaciones.(Warnes, Little, and Keevil 2015)

La determinación de estas capacidades personales de adaptación y flexibilidad de trabajo en situaciones riesgosas son importantes para estructurar las dimensiones de los protocolos de actuación individual y grupal que exige la legislación ecuatoriana en el ámbito de la Seguridad e Higiene Ocupacional y sus programas de riesgo psicosocial en estas instituciones de atención de salud. (Ceular-Villamandos et al. 2021)

El personal sanitario después de exponerse a estas condiciones de trabajo agresivas y eventos traumáticos se ve con la necesidad de iniciar un nuevo desarrollo y es necesario reforzar estos campos de acción conociendo los elementos que inciden directamente en su capacidad de recuperarse de esta vulnerabilización. (Carmassi et al. 2020)

A pesar de la importancia que los investigadores otorgan a la resiliencia no hay asentimiento sobre el instrumento adecuado para medir este constructo multidimensional. Las métricas para determinar los factores del proceso resilientes consideran elementos activos y recursos dentro del individuo, su vida y su entorno. (Brassington and Lomas 2020)

Las escalas que se utilizan con más frecuencia en la investigación la resiliencia adulta son: a) la Escala de Resiliencia en la literatura de enfermería, b) el paquete de evaluación clínica para evaluar los riesgos del cliente y Fortalezas, c) la Escala de Resiliencia del Ego; y d) la escala de resiliencia para Adultos. (Sánchez-Teruel and Robles-Bello 2015)

Las medidas de resiliencia no han sido ampliamente validadas, ni su aplicación ha sido ampliamente documentado, pero entre estos instrumentos se ha desarrollado una escala de resiliencia muy aceptada que la de Connor-Davidson (CD-RISC) que considera elementos psicométricos aplicables a distintas poblaciones.

Vivir un trauma y no ayudar en su recuperación podría incrementar un retraso en el desarrollo y las capacidades de trabajo. Esta investigación conlleva a cuantificar los elementos psicrométricos con la prueba CD-RISC y comparar los resultados con la edad y el género para poder levantar una línea base sobre los elementos significativos a cubrir 
en los protocolos de control de riesgo psicosocial causados por COVID-19 en el personal de sanitario.

\section{Metodología}

Se utilizó un muestreo estratificado para separar la población en segmentos exclusivos (García León et al. 2018), en este caso Ambato cuenta con una población urbana con165185 habitantes y donde existe 23,44 médicos por 10000 habitantes. Para minimizar el grado de error se trabajó con toda la población de personal sanitario. Se determinó un tamaño de muestra de 76 individuos calculados con un $95 \%$ de confiabilidad y un $10 \%$ de error (Duffau T. 1999), los datos fueron generados directamente del personal de salud, quienes utilizaban una encuesta desarrollada en el Google forms.(Agha et al. 2019)

\section{Determinación de la resiliencia}

Para medir la resiliencia se han desarrollado escalas específicas (Doll 2013), como la escala de resiliencia de Doll (1993) que utiliza 20 items que cubren la interacción social, habilidad para resolver discordias pequeñas, frecuencia de conductas sociales y la habilidad para solucionar conflicto de pares; la escala de(Wagnild and Young 1993) que considera las competencia personales y la aceptación de si mismo y de su vida; la escala de (Jew and Green 1998) que trabaja con las creencias optimistas en 21 items de su cuestionario.

Seguidamente (Constantine, Benard, and Diaz 1999) proponen su escala que evalúa los factores protectores internos de la resiliencia; (Arthur et al. 2007) elaboraron la herramienta Pollar consideraron un cuestionario de 114 items donde consideran factores protectores y de riesgo pero poco utilizado por los promotores de resiliencia por cargar muchas preguntas a los elementos de riesgo que a los de protección (Sarkar and Fletcher 2014). La escala de resiliencia que (Oshio et al. 2003) basan su herramienta en las habilidades y capacidades que contribuyen a disminuir la vulnerabilidad; (Perry, Bard, and Title 2000) desarrollaron su escala considerando la solución de los problemas de discapacidad, las habilidades y problemas sociales, la sensación de impotencia y las competencias de factores resilientes; (Connor and Davidson 2003).

La herramienta CD-RISC se desarrolló inicialmente como una encuesta para medir la resiliencia, considera la cualidad personal que refleja la tendencia para lograr el crecimiento cuando los factores protectores que se vuelven ineficaces y cuando la homeostasis biopsicoespiritual se interrumpe (Connor and Davidson 2003) consideran la habilidad para afrontar sus circunstancias a situaciones vitalesantes. Las dificultades que llevará a la persona a alcanzar un mayor nivel de equilibrio biopsicoespiritual $u$ homeostasis inicial. El método considera cinco factores: a.) personal competencia, altos estándares y tenacidad; b.) confianza en los instintos de uno, tolerancia de lo negativo afectar y fortalecer los efectos del estrés ; c.) aceptación positiva del cambio y seguridad relaciones; d.) control; y e.) influencias espirituales (Connor and Davidson 2003) El CD- 
RISC agrupa todas las preguntas en cuatro campos: fortaleza, optimismo, objetivos y recursos. Ver tabla 1.

Para efectuar la encuesta con el CD-RISC se desarrolló una hoja en el Google forms cuyo enlace se compartió vía WhatsApp, para evitar manipulación de documentos y reuniones de apertura y cierre por causa de la pandemia por COVID-19. El personal sanitario que respondió la encuesta forma parte de los hospitales más representativos y que son considerados de primera línea en respuesta contra el COVID-19.

Tabla 1. Cuestionario CD-RISC

\begin{tabular}{ll}
\hline $\mathrm{N}^{\text {}}$ & Pregunta \\
\hline 1 & ¿Soy capaz de adaptarme en los cambios? \\
2 & ¿Ante la adversidad busco relaciones seguras que me ayuden? \\
3 & ¿A veces el destino y Dios me pueden ayudar? \\
4 & ¿Puedo prever cosas que van a ocurrir? \\
5 & ¿El éxito pasado da la confianza para un nuevo desafío? \\
6 & ¿Miro el lado humorista de las cosas? \\
7 & ¿Enfrentarme con la tensión me hace más fuerte? \\
8 & ¿Tiendo a venirme abajo después de una adversidad? \\
9 & ¿Las cosas ocurren por una razón? \\
10 & ¿Me esfuerzo cueste lo que cueste? \\
11 & ¿Puedo alcanzar los objetivos que me proponga? \\
12 & ¿Cuándo las cosas parecen desesperadas, no me rindo? \\
13 & ¿Ante la adversidad sé cómo conseguir ayuda? \\
14 & ¿Bajo la presión, focalizo y pienso más claramente? \\
15 & ¿Prefiero tomar la delantera en la solución de un problema? \\
16 & ¿No me desanimo fácilmente ante el fracaso? \\
17 & ¿Pienso que soy una persona fuerte? \\
18 & ¿Tomo decisiones impopulares o difíciles? \\
19 & ¿Puedo manejar sentimientos desagradables? \\
20 & ¿Actúo debido a un presentimiento, sin saber por qué? \\
21 & ¿Tengo objetivos fuertes de vida? \\
22 & ¿Tengo el control de mi vida? \\
23 & ¿Me gusta el desafío? \\
25 & ¿Me esfuerzo por lograr mis objetivos? \\
\hline$N$ & ¿Estoy orgullosos de mis logros? \\
\hline
\end{tabular}

Nota: Cuestionario CD-RISC en su versión de 1992 adaptado por los autores

\section{Resultados}

\section{Resultado de resiliencia en función de la edad}

En la gráfica 1. se observa el resultado del valor de la resiliencia en función de la edad. La encuesta del CD-RISC, se adaptó en hoja de toma de datos aumentando campos de 
identificación demográfica y puesto de trabajo. Antes de su aplicación fue validada por cuatro expertos de la Universidad Técnica de Ambato, Universidad Autónoma de los Andes y Universidad Nacional de Chimborazo.

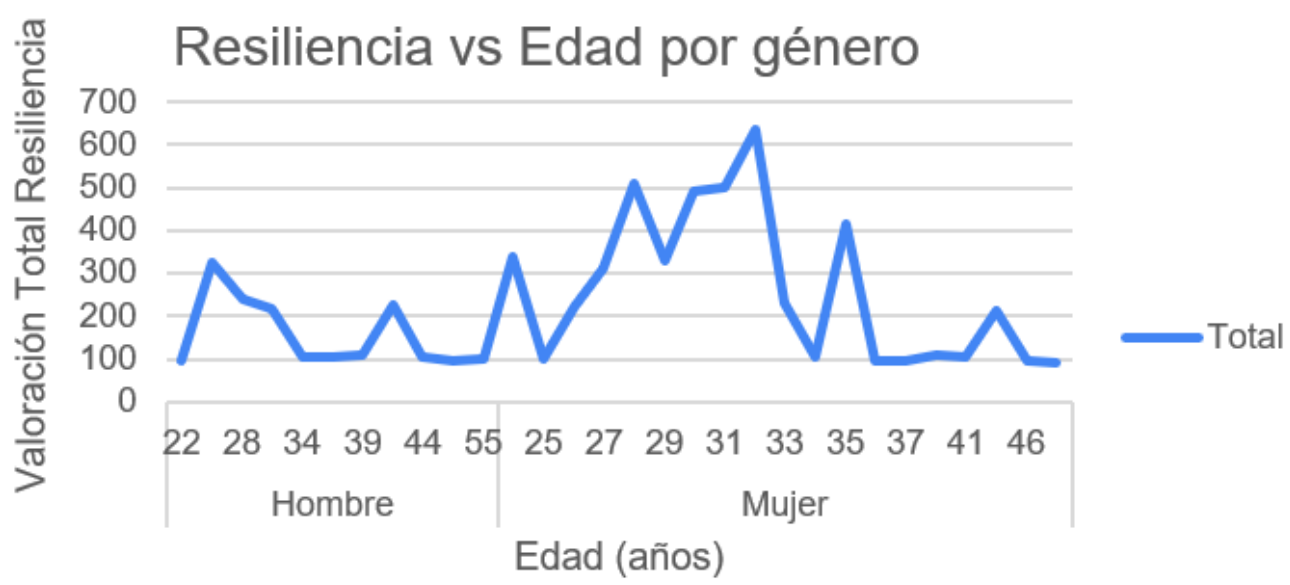

Figura 1. La curva muestra un valor de resiliencia acumulada en función de edad del personal sanitario de los hospitales de Ambato. Los rangos de edad están definidos de acuerdo con la población trabajadora de la muestra.

\section{Resultado de resiliencia por factor}

En la gráfica 2. se observa el resultado del valor de la resiliencia para cada factor individual (Fortaleza, optimismo, objetivos y recursos) sin considerar género del personal sanitario de los hospitales de la ciudad de Ambato.

gráfica 1. se observa el resultado del valor de la resiliencia en función de la edad.

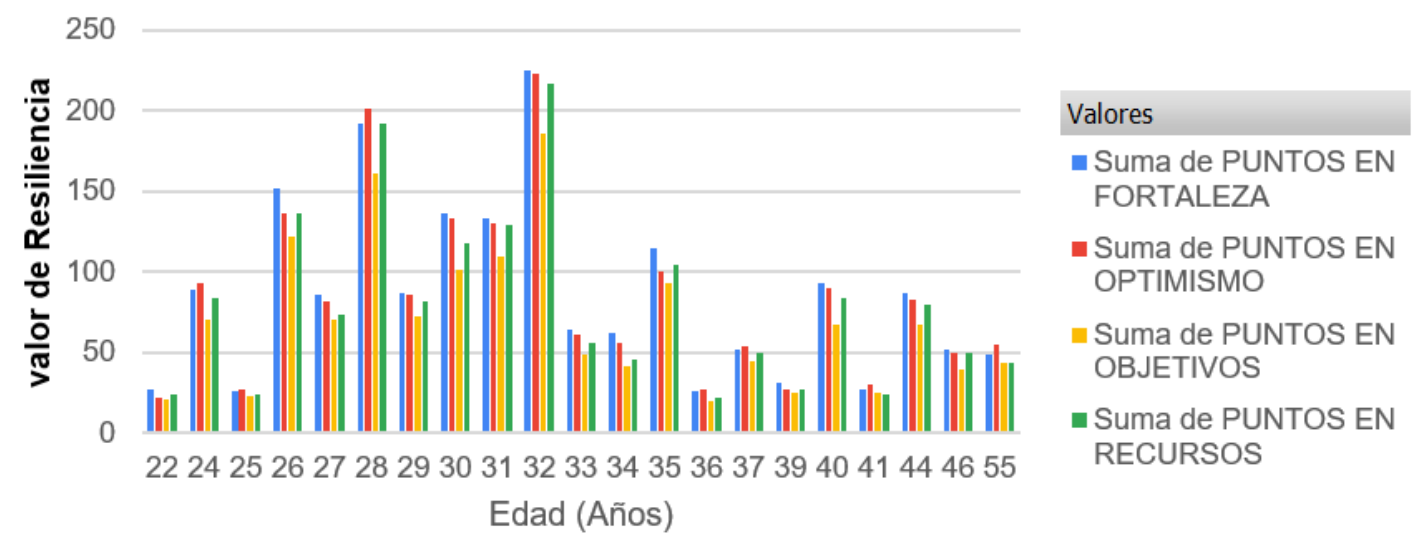

Figura 2. La gráfica muestra los valores de resiliencia para cada factor del método CD-RISC. Los rangos de edad de la muestra están definidos de acuerdo con la población trabajadora encuestada. 


\section{Conclusiones}

- Según valores obtenidos con el CD-RISC para una muestra de 76 personas que trabajan en primera línea manejando pacientes con COVID-19 se determinó que el valor más alto de nivel de resiliencia la tiene el personal sanitario de género femenino con un valor de 632 a la edad de 32 años. Según la gráfica 1 . se encontró el nivel de resiliencia más alto para el género masculino con un valor de 327 a una edad de 26 años. Así mismo se determinaron los niveles más altos de Fortaleza, Optimismo, Objetivos y Recursos con valores de: 225, 223, 186 y 217 respectivamente a los 32 años.

- El nivel de resiliencia más alto para el personal sanitario de la ciudad de Ambato la tienen la población femenina a la edad de 32 años superando al personal masculino en un $51,57 \%$.

\section{Referencias}

Abdullahi, Leila, John Joseph Onyango, Carol Mukiira, Joyce Wamicwe, Rachel Githiomi, David Kariuki, Cosmas Mugambi, Peter Wanjohi, George Githuka, Charles Nzioka, Jennifer Orwa, Rose Oronje, James Kariuki, and Lilian Mayieka. 2020. "Community Interventions in Low-And Middle-Income Countries to Inform COVID-19 Control Implementation Decisions in Kenya: A Rapid Systematic Review" edited by F. Di Gennaro. PLOS ONE 15(12):e0242403. doi: 10.1371/journal.pone.0242403.

Agha, Riaz, Ali Abdall-Razak, Eleanor Crossley, Naeem Dowlut, Christos Iosifidis, and Ginimol Mathew. 2019. "STROCSS 2019 Guideline: Strengthening the Reporting of Cohort Studies in Surgery." International Journal of Surgery 72:156-65. doi: 10.1016/j.ijsu.2019.11.002.

Arthur, Michael W., John S. Briney, J. David Hawkins, Robert D. Abbott, Blair L. Brooke-Weiss, and Richard F. Catalano. 2007. "Measuring Risk and Protection in Communities Using the Communities That Care Youth Survey." Evaluation and Program Planning 30(2):197-211. doi: 10.1016/j.evalprogplan.2007.01.009.

Brassington, Kate, and Tim Lomas. 2020. "Can Resilience Training Improve Well-Being for People in High-Risk Occupations? A Systematic Review through a Multidimensional Lens." Journal of Positive Psychology. doi: 10.1080/17439760.2020.1752783.

Carmassi, Claudia, Claudia Foghi, Valerio Dell'Oste, Annalisa Cordone, Carlo Antonio Bertelloni, Eric Bui, and Liliana Dell'Osso. 2020. "PTSD Symptoms in Healthcare Workers Facing the Three Coronavirus Outbreaks: What Can We Expect after the COVID-19 Pandemic." Psychiatry Research 292:113312. doi: 10.1016/j.psychres.2020.113312.

Cavalcanti, Yuri Wanderley, Rennis Oliveira da Silva, Leonardo de Freitas Ferreira, Edson Hilan Gomes de Lucena, Andreza Maria Luzia Baldo de Souza, Denise de Fátima Barros Cavalcante, Marcelo de Castro Meneghim, and Antonio Carlos 
Pereira. 2020. "Economic Impact of New Biosafety Recommendations for Dental Clinical Practice during Covid-19 Pandemic." Pesquisa Brasileira Em Odontopediatria e Clinica Integrada 20:1-9. doi: 10.1590/pboci.2020.143.

Ceular-Villamandos, Nuria, Virginia Navajas-Romero, Lorena Caridad y López del Río, and Lucia Zita Zambrano-Santos. 2021. "Workplace Situation and Well-Being of Ecuadorian Self-Employed.” Sustainability 13(4):1892. doi: 10.3390/su13041892.

Connor, Kathryn M., and Jonathan R. T. Davidson. 2003. "Development of a New Resilience Scale: The Connor-Davidson Resilience Scale (CD-RISC).” Depression and Anxiety 18(2):76-82. doi: 10.1002/da.10113.

Constantine, Norman A., Bonnie Benard, and Marycruz Diaz. 1999. Measuring Protective Factors and Resilience Traits in Youth: The Healthy Kids Resilience Assessment.

Doll, Beth. 2013. "Enhancing Resilience in Classrooms." Pp. 399-409 in Handbook of Resilience in Children: Second Edition. Springer US.

Duffau T., Gastón. 1999. “Tamaño Muestral En Estudios Biomédicos.” Revista Chilena de Pediatría 70(4):314-24. doi: 10.4067/s0370-41061999000400009.

García León, Maria Ángeles, Andrés González-Gomez, Humbelina Robles-Ortega, Jose Luís Padilla, and Isabel Peralta-Ramirez. 2018. "Propiedades Psicométricas de La Escala de Resiliencia de Connor y Davidson (CD-RISC) En Población Española." Anales de Psicología 35(1):33-40. doi: 10.6018/analesps.35.1.314111.

Jew, Cynthia L., and Kathy E. Green. 1998. "Effects of Risk Factors on Adolescents' Resiliency and Coping." Psychological Reports 82(2):675-78. doi: 10.2466/pr0.1998.82.2.675.

Oshio, Atsushi, Shinji Nagamine, Hitoshi Kaneko, and Motoyuki Nakaya. 2003. "Construct Validity of the Adolescent Resilience Scale." Psychological Reports 93(3 II):1217-22. doi: 10.2466/pr0.2003.93.3f.1217.

Perry, Author, Joseph D.;. Bard, and E. M. Title. 2000. Resilience Revisited: Urban Children Assessment and Intervention. Construct Validity of a Resilience Profile for Students with Disabilities (Resilience Subdomains of Coping with Disability ScalesParent Rating Scale).

Sánchez-Teruel, David, and María Auxiliadora Robles-Bello. 2015. 4-Item Resilience Scale (RS-14): Psychometric Properties of the Spanish Version Escala de Resiliencia 14 Ítems (RS-14): Propiedades Psicométricas de La Versión En Español. Vol. 2.

Sarkar, Mustafa, and David Fletcher. 2014. "Psychological Resilience in Sport Performers: A Review of Stressors and Protective Factors." Journal of Sports Sciences 32(15):1419-34. doi: 10.1080/02640414.2014.901551.

Wagnild, GM, and H. Young. 1993. "Development and Psychometric." Journal of Nursing Measurement 1(2):165-178.

Warnes, Sarah L., Zoë R. Little, and C. William Keevil. 2015. "Human Coronavirus 229E 
Remains Infectious on Common Touch Surface Materials." MBio 6(6). doi: 10.1128/mBio.01697-15.

Yin, Qianlan, Zhuoer Sun, Tuanjie Liu, Xiong Ni, Xuanfeng Deng, Yanpu Jia, Zhilei Shang, Yaoguang Zhou, and Weizhi Liu. 2020. "Posttraumatic Stress Symptoms of Health Care Workers during the Corona Virus Disease 2019." Clinical Psychology \& Psychotherapy 27(3):384-95. doi: 10.1002/cpp. 2477.

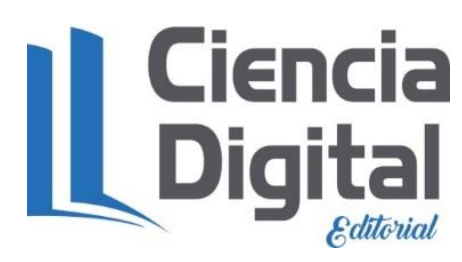




\section{PARA CITAR EL ARTÍCULO INDEXADO.}

Gordón Villalba, P. del R., Córdova Suárez, M. A., Villacres Cevallos, E. P., \& Barreno Avila, E. M. (2021). La edad y la resiliencia en el personal de salud que atiende pacientes con Covid-19. Caso de la ciudad de Ambato. Anatomía Digital, 4(2), 122-131. https://doi.org/10.33262/anatomiadigital.v4i2.1669

\section{Liencia}

El artículo que se publica es de exclusiva responsabilidad de los autores y no necesariamente reflejan el pensamiento de la Revista Anatomía Digital.

El artículo queda en propiedad de la revista y, por tanto, su publicación parcial y/o total en otro medio tiene que ser autorizado por el director de la Revista Anatomía Digital.
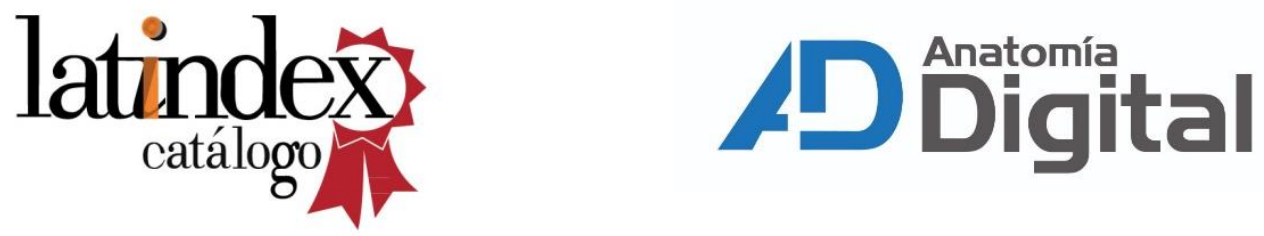